\title{
Database for vegetable phytochemicals and their mechanism of action
}

\author{
Sundararajan Kriushnapriya ${ }^{1 *}$, Kalidass Dhinagar ${ }^{2}$, Subramanian Malathy ${ }^{1}$ \& Kaniappan \\ Mani $^{3}$
}

1Department of Botany, PSGR Krishnammal College for Women, Coimbatore, TamilNadu - 641004, India; ${ }^{2}$ Department of Computer Science; PSG College of Arts and Science, Coimbatore, TamilNadu - 641014, India; 3 Department of Botany, PSG College of Arts and Science, Coimbatore, TamilNadu - 641014, India; Sundararajan Kriushnapriya - E mail: kriush.sundar@gmail.com; * Corresponding author

Received May 18, 2012; Accepted May 24, 2012; Published May 31, 2012

\begin{abstract}
:
In an endeavor to screen bioactive compounds present in vegetables with effective mechanism using in silico method lead us to develop a vegetable phytochemicals and their target database (VPTD). The VPTD is a unique bioinformatics resource that compiles information about phytochemicals from vegetables and their mechanism. VPTD contains 2496 phytochemicals from 27 vegetables, their 3D images and their 1337 possible biological mechanism. Each phytochemical contain records of seven data fields providing detailed information on name, source, amount present, structure and mechanistic information. This information has been manually extracted and manually verified from numerous sources, including other electronic databases, textbooks and scientific journals. VPTD is fully searchable and supports extensive text search. The main focus of the VPTD is on providing possible mechanism of phytochemicals, which will help in discovery of potential drugs from one of the common bioresource-vegetable. VPTD is freely available.
\end{abstract}

Availability: http://www/vptd.in.

Key words: Vegetable, Target, Database, Phytochemical, PASS

\section{Background:}

Chemical constituents of vegetables are known to be responsible for the beneficial health effects, namely vitamins, minerals, trace elements, dietary fiber, proteins and especially a wide variety of phytochemicals. Though a great deal of information has been acquired about phytochemicals and their role over the past few decades, much of this information is highly dispersed and of limited accessibility, often being confined to specialized textbooks, obscure government documents or subscription-requiring scientific journals. Fortunately, over the past few years a number of web-accessible databases have started to appear, such as the pubchem, chembank, Dr. Dukes phytochemical database and so on. They focus on providing information on either chemical property, structure, ambiguous mode of action or only about the source of phytochemicals. A few databases were reported for biological applications, which are mostly focusing on particular aspects of dietary phytochemicals [1-3], which are based on experimental and published secondary data from the scientific reports. However, they lack information on phytochemicals along with its unrevealed possible mechanism of actions. In an effort to integrate informations about phytochemicals as well as their targets, this Vegetable Phytochemicals and phytochemical Target Database (VPTD) were developed to be useful in finding effective chemical entities in drug discovery. 


\section{Methodology:}

Data on phytochemicals were manually collected from online databases [4-6] and from peer-reviewed scientific articles [7-12]. From 27 commonly consumed vegetables in India, 2496 phytochemicals were collected and this data was compiled using Microsoft Access ${ }^{\circledR}$ 2003. The 3D molecular structures of the phytochemicals were rendered from online small molecular databases namely, Pubchem and Chembank as 2D structures as .sdf file. For those, whose structures are not available in the online databases were drawn using ACDLab's Chemsketch 12.0 version [13], 3D optimized and energy minimized using CHARM force field and saved as .mol file. They were submitted to PASS (Prediction of Activity Spectra for Substances) to predict the possible biological activity of phytochemicals [14]. PASS has provided possible mechanisms with statistical probability as - probable activity (pa), probable inactivity (pi).

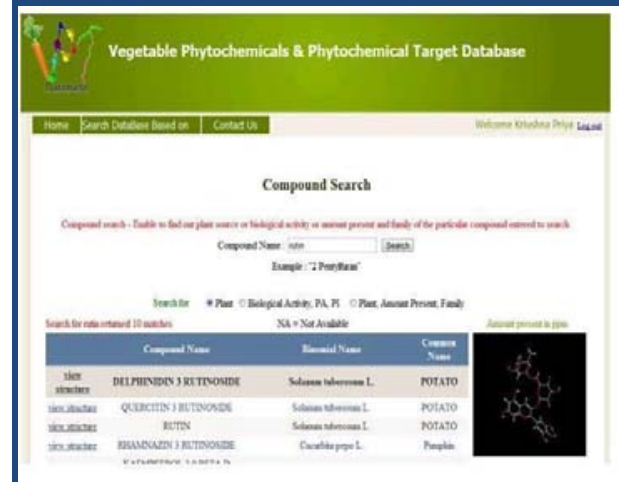

a)

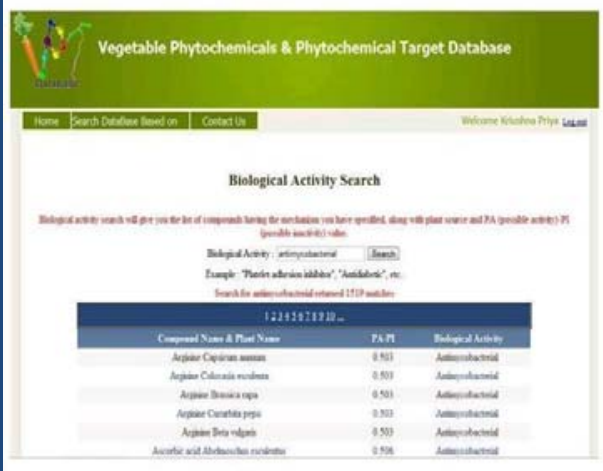

d)

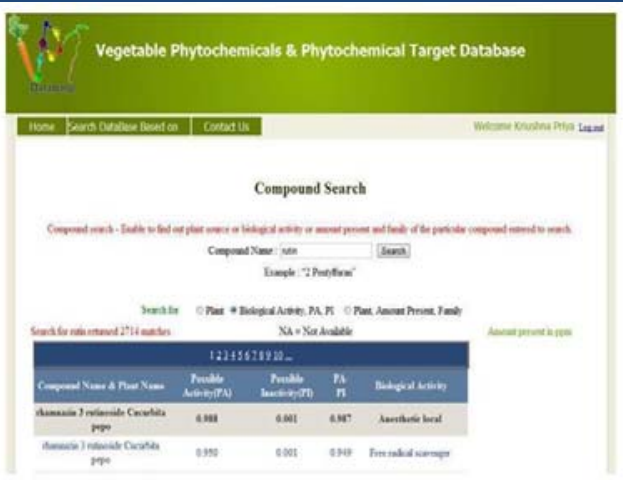

b)

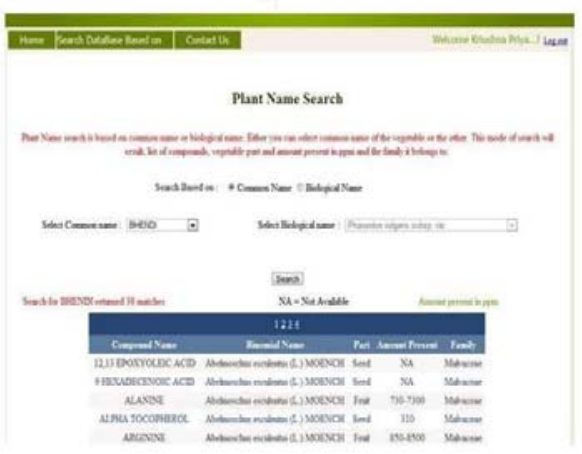

e)
The activity index (Pa-Pi) for each phytochemical was manually calculated. PASS prediction for each vegetable phytochemical was saved as .xls files. VPTD was developed using Microsoft Access ${ }^{\circledR}$ 2003. This database comprises primary Table and secondary Table 1 (see supplementary material). Primary (Table 1) consists of seven data fields (name of the phytochemical, structure, source common name, source binomial, and source family and part of the plant body). Secondary (Table 1) contains four data fields (pa; pi; pa-pi and biological activity). Compound name_source binomial (E.g. Betanin_Beta vulgaris) was set as primary key in the database. Finally, data were exported from Microsoft Access ${ }^{\circledR}$ to Microsoft SQL server 2005, which is used as the web interface. The front end of the database was designed using Microsoft .NET Framework SDK v2.0.

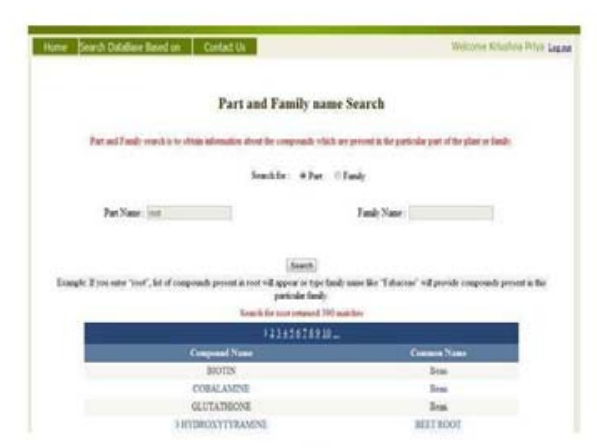

g)

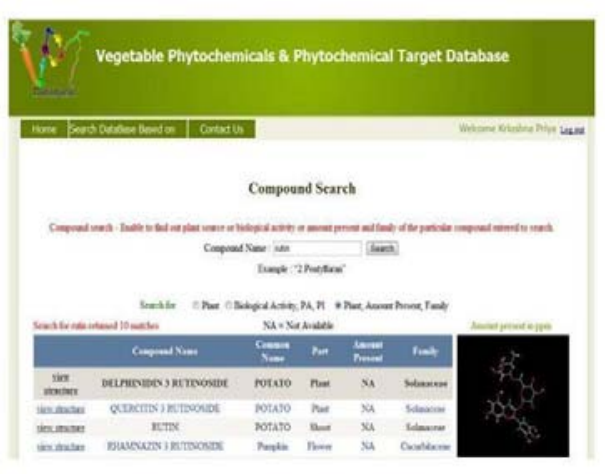

c)

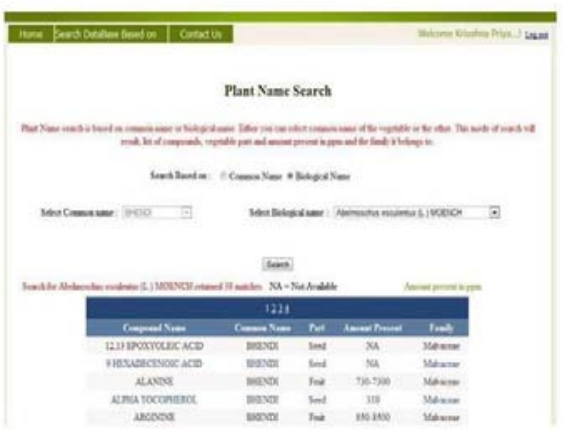

f)

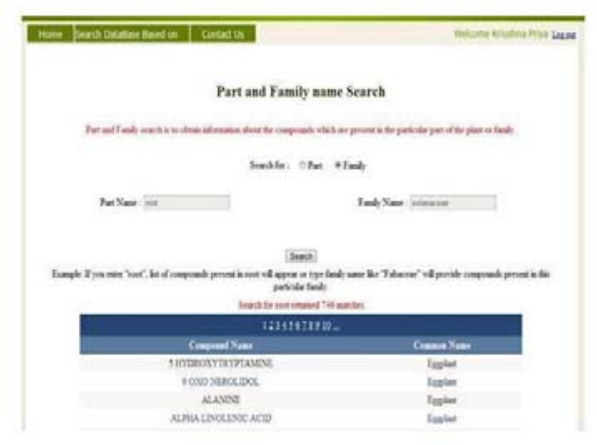

h)

Figure 1 : a) The output of compound search - plant category; b) The output of compound search - biological activity category; c) The output of compound search - plant, amount present \& family category; d) The output of biological activity search category; e) The output of plant name search category for common name field; f) The output of plant name search category for binomial name field; g) The output of part and family name search category for part of plant field; h) The output of part and family name search category for family name field 


\section{VPTD features:}

VPTD has a web interface at http:/ / www.vptd.in/. It currently abodes 1337 mechanism of actions of 2496 phytochemicals present in 27 commonly consumed vegetables in India. It also provides name of the phytochemicals, their source binomial and common name, part of the plant body (ppm). Table 1 gives the different search modes and examples of typical search terms of the VPTD. The output of "compound search-plant" category is illustrated in (Figure 1a), which is obtained by giving an input "Rutin." The result contains plant source of the compound, Source binomial, source common name and structure. Similarly for the same input in "compound searchbiological activity" category (Figure 1b), the output includes compound name with source binomial, biological activity, pa value, pi value and pa-pi value. For the same input in "compound search-plant, amount present \& family" category, the output (Figure 1c) includes source common name, plant part, amount present (ppm), plant family and structure. In "biological activity search" category, for the input "antimycobacterial," the output (Figure 1d) contains the list of all possible activities with pa value, pi value and pa-pi value. In "plant name search" category by selecting the common name of the plant from dropdown box (e.g. Bhendi) the output includes a list of compounds present in that vegetable along with the details-source binomial, part of the plant, amount present and family (Figure 1e). If the binomial name is selected from dropdown box the output will be same by replacing binomial with common name (Figure 1f). In the "part and family name search" category, for the input "root" in part of plant field, the output contains the compounds present in the root part with their source common name (Figure 1g). In the same search category, for the input "Solanaceae" in family name field the output comprised of compounds belongs to the input family along with the source common name (Figure 1h). Queries are text based and case insensitive.
Utility:

VPTD is useful in scrutinizing the vegetable leads based on their predicted biological activity, which will reduce the time involved in various types of in vitro experiments. With the aid of VPTD, a vegetable lead can be proved for its specific pharmacodynamic property by conducting precise wet lab tests, which is less time consuming and cost effective. Thus VPTD is a specific database designed for vegetables and will be useful in facilitating the identification and exploration of potential therapeutic targets of vegetable phytochemicals.

\section{Caveat and Future Developments:}

The database website has a provision for updating and adding new entries (as .xls files or even a folder containing .xls files) in future by the database manager. All data fields will be curated and then accepted by the built in program. Database will be extended for phytochemicals present in spices and fruits.

\section{References:}

[1] Nirmala Arulrayan et al. Bioinformation 2007 2: 22 [PMID: PMID: 18084646]

[2] Christine Hoefkens et al. Food Chemistry. 2009 113: 799 [PMID: 20691244]

[3] Neveu V et al. Database 2010 1: 9 [PMID: 20428313]

[4] www.emi.gov-drdukes.com

[5] www.pubchem.ncbi.nlm.nih.gov

[6] www.chembank.broadinstitute.org

[7] www.thieme-connect.de/ejournals/toc/plantamedica

[8] www.chromatographia.de

[9] www.nutritionsociety.org

[10] www.journalarchive.jst.go.jp/english/jnltop_en.php

[11] www.elsevier.com/locate/phytochem

[12] www.elsevier.com/locate/foodchem

[13] www.acdlabs.com/chemsketch

[14] www.ibmc.msk.ru/PASS/

Edited by P Kangueane

Citation: Kriushnapriya et al. Bioinformation 8(10): 492-495 (2012)

License statement: This is an open-access article, which permits unrestricted use, distribution, and reproduction in any medium, for non-commercial purposes, provided the original author and source are credited 


\section{Supplementary material:}

Table 1: Categories of typical search terms of VPTD

\begin{tabular}{|c|c|c|c|}
\hline Search category & Query type & Search term & Example input \\
\hline Compound & $\begin{array}{l}\text { Plant Biological activity } \\
\text { Plant part, amount present } \\
\text { and family }\end{array}$ & Compound name & Rutin \\
\hline Biological activity & Activity & Name of the activity & Antimycobacterial \\
\hline Plant & Common name or binomial & $\begin{array}{l}\text { Selection of either common name or binomial } \\
\text { from drop down box }\end{array}$ & Bhendi or Phaseolus vulgaris \\
\hline $\begin{array}{l}\text { Plant part and } \\
\text { family }\end{array}$ & Part of the plant or family & Either name of the plant part or family & Root or Solanaceae \\
\hline
\end{tabular}

